\section{Theoretical studies of core excitation and ionization in molecular systems}

\author{
Ping Lin and Robert R. Lucchese*
}

\author{
Department of Chemistry, Texas A\&M University, PO Box \\ 30012, College Station, TX 77842-3012, USA. \\ E-mail: lucchese@mail.chem.tamu.edu
}

Photoionization cross sections have been computed using correlated initial and final target states obtained with standard $a b$ initio techniques and using a numerical representation of the continuum scattering orbitals. Here, core ionization of small molecular systems will be focused on. A brief review of previous results obtained using these methods is given. New results are then given for the $\mathrm{N} 1 s$ ionization of $\mathrm{NO}$ and the $\mathrm{N} 1 s$ ionization of $\mathrm{N}_{2}$. In the study of $\mathrm{NO}$, the spin-coupling scheme which best describes the shape-resonant state and the energy dependence of the branching ratios for the singlet and triplet final ion states will be considered. In the study of $\mathrm{N}_{2}$ core ionization, the effects of the coupling of nearly degenerate core levels and the effects of correlation on the importance of interchannel coupling will be considered.

Keywords: molecules; photoionization; core ionization.

\section{Introduction}

Much progress has been made in the ability to compute the photoionization cross sections of molecules. For linear molecules the azimuthal symmetry reduces the difficulty of the calculation. Thus for these systems it is possible to perform very accurate calculations including both correlation in the initial and ionized target states and correlation of the photoelectron with the target electrons. In this paper we will discuss results obtained with the multichannel Schwinger configuration interaction method (MCSCI) (Stratmann \& Lucchese, 1995) which we have used to study a number of core ionization processes. To consider larger systems, simpler treatments of the correlation effects are usually employed.

There are a number of interesting resonance effects which occur at energies near the threshold for core ionization of a molecule (Chen et al., 1989). Just below the threshold for ionization there are transitions from the core level to valence and Rydberg levels of the molecule. These states then have a number of modes of decay. The excited states can decay by emission of light, by an Auger-type decay process, and by fragmentation. The Auger process is known as a resonant Auger decay. Furthermore, there are two modes of non-radiative decay in the resonant Auger process. The first mode is a participatortype decay where the electron in the valence or Rydberg level participates in the decay process; the second mode is a spectator-type decay where the initially excited electron remains in its excited state and the other electrons take part in the decay process. In our study on the resonant Auger ionization of $\mathrm{CO}$ due to the $\mathrm{C} 1 s \rightarrow 2 \pi^{*}$ excitation, we found that the MCSCI approach can obtain the width of the resonant state and can predict the degree to which the decay process is of the participator or spectator type (Botting \& Lucchese, 1997).

When the photon energy is greater than the threshold for the core ionization process, the resonances that occur are qualitatively very similar to those found in photoionization from the valence shell. One finds one-electron resonances that are due to angular momentum barriers. These resonances are usually fairly broad when compared with two-electron resonances. Often these shape resonances resemble unoccupied valence antibonding orbitals as in the photoionization of CO (Lucchese \& McKoy, 1983) or $\mathrm{N}_{2}$ (Lucchese et al., 1982) in which there are prominent $\sigma^{*}$-type resonances which are trapped by an $l=3$ angular momentum barrier. However, we have found in the photoionization of S $1 s$ in $\mathrm{SF}_{6}$ that there is a resonance of $t_{1 u}$ symmetry which is not similar to any of the unoccupied valence orbitals of that system and is characterized by an $l=9$ angular momentum barrier (Natalense \& Lucchese, 1999).

One difference between valence ionization and core ionization is that generally in the valence region there are several valence ion states that are well described by single-hole states. At higher energies in the valence region there are usually shake-up states that can be characterized by two vacancies in the bound orbitals of the initial state and one virtual orbital occupied. These states often strongly mix with the inner valence single-hole states, leading to strong configuration mixing effects that can complicate the interpretation of the photoionization cross sections. In the region of core ionization there are three possible cases which can occur. If there is only one atom in the molecule of a particular type, then in the region of the core ionization of that atom there will be only one hole state. If there are two or more atoms of a particular type, and if the atoms are equivalent, there will be a number of degenerate or nearly degenerate hole states. In these cases, there can be interesting coupling effects between the hole states and the vibrational motion of the molecule. One such effect is dynamic hole localization (Domcke \& Cederbaum, 1977). If the atoms are not equivalent, then there will be a more substantial splitting between the core levels.

In a previous study of the $\mathrm{C} 1 s$ photoionization of acetylene (Lin \& Lucchese, 2000a), we have considered the case of chemically equivalent atoms. In that study we considered the effects of electronelectron correlation on the description of the $\sigma^{*}$ shape resonance which occurs at about $20 \mathrm{eV}$ above the $\mathrm{C} 1 \mathrm{~s}^{-1}$ threshold. It was found that, without the inclusion of correlation in the description of the target states, the effects of interchannel coupling were exaggerated. We further found quantitative agreement with experimental cross sections (Thomas et al., 1999) for the resolved $1 \sigma_{g}^{-1}$ and $1 \sigma_{u}^{-1}$ partial photoionization cross sections with a calculation including the two hole states and the four strongest shake-up states.

\section{Computational methods}

The method we have used to study the various photoionization processes is the MCSCI method. In this method, the close-coupling approximation is used with the target states represented by configuration interaction $(\mathrm{CI})$ wave functions. This leads to a set of coupled integro-differential equations that are solved using the Schwinger variational method. This method has been described in detail elsewhere (Lucchese et al., 1986; Bandarage \& Lucchese, 1993; Stratmann \& Lucchese, 1995; Stratmann et al., 1996) so we will only give a brief outline of the method here. In the MCSCI method, the photoionized state of a molecule is described by a stationary state wave function of the form

$$
\Psi_{s}=\sum_{p} \Phi_{p}\left(\chi_{p}\right)=\sum_{p} \sum_{\mu} C_{p \mu} \psi_{\mu}\left(\chi_{p}\right)
$$

where $\chi_{p}$ is the one-electron scattering wave function of channel $p$. $\Phi_{p}$ represents the CI target ion wave function of channel $p$, which is a linear combination of spin-adapted $N-1$ electron configuration state functions (CSF) $\psi_{\mu} . \Phi_{p}\left(\chi_{p}\right)$ and $\psi_{\mu}\left(\chi_{p}\right)$ are antisymmetrized 
spin- and symmetry-adapted products of the corresponding $N-1$ electron functions and the continuum one-electron function $\chi_{p}$.

The wave function $\Psi_{s}$ satisfies the time-independent Schrödinger equation

$$
\left(H_{N}-E\right)\left|\Psi_{s}\right\rangle=0,
$$

with the appropriate asymptotic boundary condition and where $H_{N}$ is the $N$-electron Hamiltonian and $E$ is the total electronic energy. Given the molecular orbitals $\left\{\varphi_{i}\right\}$ and the CI wave functions of the residual ion of different channels, we are able to obtain the coupled variational equations for the scattering wave functions,

$$
\left\langle\delta \chi_{p}\left|\left[h_{p}-\varepsilon_{p}\right]\right| \chi_{p}\right\rangle+\sum_{q}\left\langle\delta \chi_{p}\left|U_{p q}\right| \chi_{q}\right\rangle=0
$$

where $p, q$ are the channel indexes,

$$
h_{p}=-(1 / 2) \nabla_{p}^{2}-\sum_{\alpha}\left(Z_{\alpha} / r_{p \alpha}\right)
$$

$\varepsilon_{p}$ is the asymptotic kinetic energy of the photoelectron in channel $p$, with $\varepsilon_{p}=E-E_{p}$, where $E_{p}$ is the electronic energy of the residual ionic state in channel $p$, and $U_{p q}$ is the non-local optical potential (Bandarage \& Lucchese, 1993).

The Lippmann-Schwinger equation is then

$$
\bar{\chi}=\bar{\chi}^{0}+\underline{G}_{c} \underline{V}_{Q} \bar{\chi},
$$

where $\bar{\chi}$ and $\bar{\chi}^{0}$ are the vectors of the channel-scattering wave function and Coulomb wave function, respectively. The potential $\underline{V}_{Q}$ in equation (5) is a Phillips-Kleinman pseudopotential (Lucchese et al., 1982; Bandarage \& Lucchese, 1993), which enforces the orthogonality between a given scattering wave function and selected molecular orbitals. In (5), $\underline{G}_{c}$ is the multichannel Coulomb Green's function matrix given by

$$
\left(\underline{G}_{c}\right)_{p q}=G_{c}\left(\varepsilon_{p}\right) \delta_{p q} .
$$

The variational functional for the photoionization transition moment leading to the target state of channel $p$ can be constructed as

$$
\begin{aligned}
I_{p}^{L(V)}= & \left\langle R_{p}^{L(V)} \mid \chi_{p}^{0}\right\rangle+\sum_{\alpha, \beta}\left\langle R_{p}^{L(V)}\left|\underline{G}_{c} \underline{V}_{Q}\right| \varphi_{\alpha}^{\prime}\right\rangle \\
& \times\left\langle\underline{V}_{Q}-\underline{V}_{Q} \underline{G}_{c} \underline{V}_{Q}\right\rangle_{\alpha \beta}^{-1}\left|\varphi_{\beta}^{\prime}\right| \underline{V}_{Q}\left|\chi_{p}^{0}\right\rangle,
\end{aligned}
$$

where the quantity $R_{i}^{L(V)}$ is a function of the electronic dipole operator, and $\left\{\varphi_{i}^{\prime}\right\}$ forms the multichannel scattering basis set. The superscript $L(V)$ denotes that this equation may be solved using either the length or the velocity approximation. This multichannel Schwinger result is further refined using Padé approximates to $I_{p}^{L(V)}$ (Lucchese \& McKoy, 1983).

The double-differential photoionization cross section of a given channel is expressed in length (or velocity) and the mixed form as

$$
\frac{\mathrm{d}^{2} \sigma_{p}^{L(V)}}{\mathrm{d} \Omega_{\hat{k}_{p}} \mathrm{~d} \Omega_{\hat{n}}}=\frac{4 \pi^{2} \hbar \omega}{c}\left|I_{p}^{L(V)}\right|^{2},
$$

and

$$
\frac{\mathrm{d}^{2} \sigma_{p}^{M}}{\mathrm{~d} \Omega_{\hat{k}_{p}} \mathrm{~d} \Omega_{\hat{n}}}=\frac{4 \pi^{2} \hbar \omega}{c} \operatorname{Re}\left[\left(I_{p}^{L}\right)^{*} I_{p}^{V}\right] .
$$

The final cross sections and the asymmetry parameters are obtained by integrating over all orientations of the molecule in the laboratory frame.

The calculation of the photoionization cross section begins with the calculations of the initial and target states. In order to simplify the calculation, we use the same set of molecular orbitals in the CI calculations of the initial and all final states. For the ionization of the core levels, these common orbitals are usually obtained by a complete-active-space self-consistent-field (CASSCF) calculation on the ground state of the molecule of interest using the MOLPRO program (Werner \& Knowles, 1996). However, for valence ionization we have also found that a state-averaged multi-configuration selfconsistent-field (MCSCF) calculation on a selection of important ion states has yielded very good results (Wells \& Lucchese, 1999). Molecular natural orbitals generated from this step are used by the MCSCI code to construct the CSFs and total electron wave functions.

In the following steps, the single center expansion (SCE) technique and numerical grid method have been employed in all integrations.

Different levels of the MCSCI calculations are performed to investigate how the different types of correlation contribute to the photoionization process. Calculations based on the single-channel Schwinger Hartree-Fock (SCSHF) approximation contain no correlation. Multichannel Schwinger Hartree-Fock (MCSHF) approximation calculations show the results of target response to the photoelectron without including target correlation. Single-channel Schwinger configuration interaction (SCSCI) calculations show the effects of target correlation. The full MCSCI calculation has all correlation effects included. In addition, by selectively including and removing channels it is possible to consider which channels are important in various resonant features. In core ionization we are particularly interested in the effects of interchannel coupling between core-hole channels and in the effects of interchannel coupling between core-hole and shake-up channels. In the study of linear systems, the full MCSCI calculation can be performed with up to $\sim 20$ ion states included in the close coupling expansion. For non-linear systems, we are only able to treat the photoionization problem at the SCSHF level. At this level, interesting shape resonance effects can be considered (Natalense \& Lucchese, 1999); however, two-electron resonance processes such as autoionization cannot be studied.

\section{Results}

\section{1. $N$ 1s ionization of NO}

In our study of the photoionization of NO, the equilibrium structure of molecular NO is used in the calculations, in which $R_{\mathrm{N}-\mathrm{O}}=$ $1.15077 \AA$. In the first step, a restricted Hartree-Fock (RHF) calculation is performed on the ion ground state using the MOLPRO program (Werner \& Knowles, 1996), followed by an MCSCF calculation including all core and valence orbitals. Dunning's aug-cc-pVTZ basis set with augmented diffuse functions was used in these calculations (Dunning, 1989). The natural orbitals are constructed from the MCSCF calculation and serve as the molecular basis set in the following MCSCI calculation.

In the next step, initial molecular states and target ion states are obtained by full CI (FCI) calculation with the graphical unitary group approach (GUGA) (Hinze, 1981) using the natural orbital set. SCSCI and two-channel Schwinger configuration interaction (2CSCI) calculations were performed using the initial and target states calculated above. The continuum scattering orbitals are obtained by performing a scattering calculation on a model potential using a set of general Gaussian-type orbitals and selecting a compact basis set from the final eigenfunctions (Stratmann et al., 1996).

The ground state of NO has the configuration of $1 \sigma_{\mathrm{O}}^{2} 2 \sigma_{\mathrm{N}}^{2} 3 \sigma^{2} 4 \sigma^{2} 5 \sigma^{2} 1 \pi^{4} 2 \pi^{1}$. The N $1 s$ ionization of NO leads to two $\Pi$ states with different multiplicity, singlet or triplet states. The calculated ionization potential (IP) is $409.59 \mathrm{eV}$ for the ${ }^{3} \Pi$ state, and $410.44 \mathrm{eV}$ for the ${ }^{1} \Pi$ state. The experimentally determined IPs are 410.3 and $411.8 \mathrm{eV}$, respectively (Siegbahn et al., 1969). The SCSCI 
and 2CSCI calculations produce very similar shapes of the total and partial cross sections. The partial cross sections from the 2CSCI calculation are plotted in Fig. 1(a). Sharp resonances occur in both channels but at slightly different positions. The maximum of the cross section is $413.5 \mathrm{eV}$ in the channel leading to the ${ }^{1} \Pi$ state and $414.5 \mathrm{eV}$ in the channel leading to the ${ }^{3} \Pi$ state. These two shape resonances are also observed in the measured photoionization cross sections for the two channels, but are broader than those we obtained (Ruedel \& Hergenhahn, 2000). By comparison with the single-channel scattering calculations, we observed little interchannel coupling between these two channels. The intensity ratio between these two channels is plotted as a function of photon energy in Fig. 1(b). In the high-energy limit, the intensity ratio ${ }^{3} \Pi /{ }^{1} \Pi$ is slightly higher than 3 , which matches the spin statistic ratio. Near the threshold, however, the intensity ratio changes dramatically. The minimum of the ratio is $\sim 0.88$, located at $413 \mathrm{eV}$, just around the first shape resonance. The maximum of the ratio is $\sim 5.24$, located at $415 \mathrm{eV}$, close to the shape resonance in the channel leading to the ${ }^{3} \Pi$ state. Experiments have verified the existence of the structure in the measured intensity ratio, but the oscillatory structure near the threshold is much less significant.

The shape resonances have been verified as $\sigma^{*}$-type resonances. Compared with previous calculations by Wallace et al. (1982), the positions of the shape resonances from our calculation are much closer to the threshold, and the intensity ratio is also different from the spin statistics that they used. For the $\mathrm{N} 1 s$ core ionization of NO, the couplings between core and valence orbitals are strong enough that a multiplet-averaged approach is not feasible. Our results also indicate that the couplings between continuum scattering wave function and valence orbitals are much less significant than the corevalence coupling. Thus, the shape resonances in the photoelectron scattering from the singlet or triplet ion potentials are observed in different positions. This is in contrast to the behavior of the corresponding resonance in the $5 \sigma^{-1}$ valence ionization of NO (Stratmann et al., 1996). In the valence region, the $5 \sigma-k \sigma$ coupling is much stronger than the $\mathrm{N} 1 s-k \sigma$ coupling. The consequence of this stronger coupling is that in the valence region there is only one strong shape resonance feature in the photoionization process. This resonant state then decays into the two possible asymptotic ${ }^{3} \Pi$ and ${ }^{1} \Pi$ channels. In

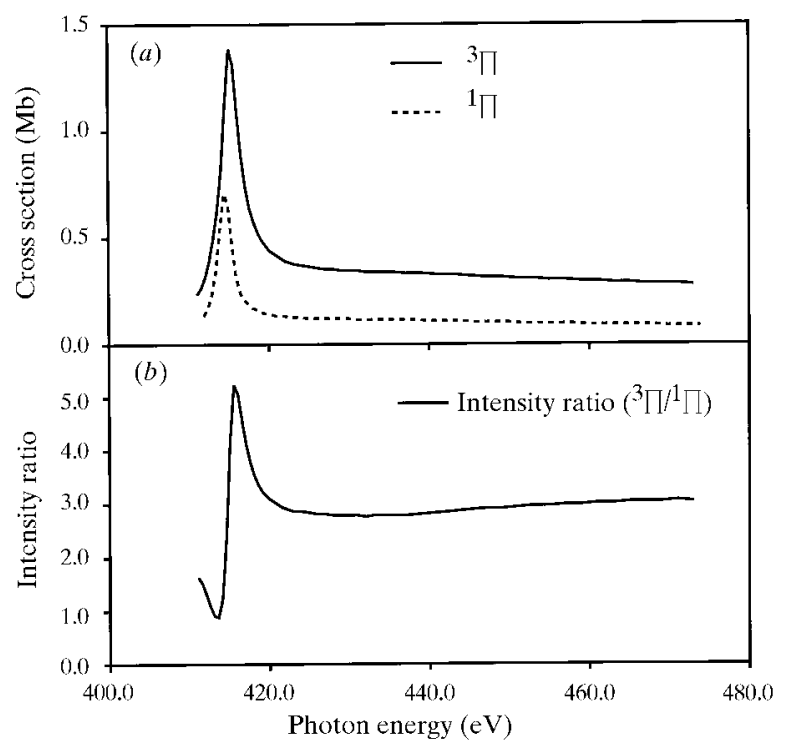

Figure 1

Photoionization cross sections for the ionization of NO leading to the $\mathrm{N} 1 s^{-1}$ ${ }^{3} \Pi$ and ${ }^{1} \Pi$ states of $\mathrm{NO}^{+}$ the partial cross sections the resonance then appears at the same energy in each channel.

The core ionization of NO is a unique system. We observed that the MCSCI calculation for this system is very sensitive to the selection of the molecular basis set. Our calculations also showed that the cross sections are very sensitive to the $\mathrm{N}-\mathrm{O}$ bond distance (Lin \& Lucchese, 2000a). This geometry dependency of the photoionization cross section may be the cause of the difference between theory and experiments.

In our current calculations, we excluded the many possible shakeup channels in the close-coupling expansion. However, we believe that the couplings between the core-hole states and shake-up channels cannot be neglected. The exclusion of the shake-up channels in the expansion may also be the cause of the disagreement.

\section{2. $N$ 1s ionization of $\mathrm{N}_{2}$}

The calculation of $\mathrm{N} 1 s$ photoionization of $\mathrm{N}_{2}$ is performed at its equilibrium geometry, in which $R_{\mathrm{N}-\mathrm{N}}=1.09768 \AA$. An RHF calculation is first performed for the ground state of the $\mathrm{N}_{2}$ molecule. A CASSCF calculation including full valence orbitals is then performed, followed by a multi-reference configuration interaction (MRCI) calculation. A set of natural orbitals is generated after the MRCI calculation. The following natural orbitals are selected to form the molecular orbital set in the selected CI calculation,

$$
1 \sigma_{g} 1 \sigma_{u} 2 \sigma_{g} 2 \sigma_{u} 3 \sigma_{g} 1 \pi_{u} 1 \pi_{g} 3 \sigma_{u} 4 \sigma_{g} 2 \pi_{u} 2 \pi_{g} 4 \sigma_{u}
$$

Two restrictions are applied in the selected CI calculation, i.e. only two electrons are allowed in the last six orbitals, and only one electron is allowed in the last four orbitals. Total and partial cross sections are obtained by coupled-channel scattering calculations.

The electronic configuration of the ground state of $\mathrm{N}_{2}$ is $1 \sigma_{g}^{2} 1 \sigma_{u}^{2} 2 \sigma_{g}^{2} 2 \sigma_{u}^{2} 3 \sigma_{g}^{2} 1 \pi_{u}^{4}$. The N $1 s$ ionization in $\mathrm{N}_{2}$ includes both $1 \sigma_{g}^{-1}$ and $1 \sigma_{u}^{-1}$ ionization channels. The calculated IP for $\mathrm{N} 1 s$ of $\mathrm{N}_{2}$ is that of the $1 \sigma_{u}^{-1}$ state, which equals $413.054 \mathrm{eV}$. The measured IP (Chen et al., 1989) is $409.938 \mathrm{eV}$. The calculated splitting between two corehole states, $1 \sigma_{u}^{-1}$ and $1 \sigma_{g}^{-1}$, is $0.144 \mathrm{eV}$. The small splitting makes it difficult to distinguish their contributions to the $\mathrm{N} 1 s$ photoionization cross section. However, the two channels did have very different dynamics in the core photoionization process due to their symmetry properties.

The total cross section of the $\mathrm{N} 1 s$ ionization and the partial cross sections of the two channels are plotted in Fig. 2, together with the total cross section measured by a high-resolution photoionization study (Kempgens et al., 1996). The experimentalists found a shape resonance at $\sim 419 \mathrm{eV}$. Many studies using a multiple-scattering model (MSM) (Dehmer \& Dill, 1975), or the Stieltjes-Tchebycheff moment theory (STMT) (Rescigno \& Langhoff, 1977), or the Schwinger method with frozen-core Hartree-Fock including core relaxation effects (RCHF) (Lynch \& McKoy, 1984), were not able to reproduce both the magnitude and position of this resonance. Our calculation found the shape resonance located at about $420 \mathrm{eV}$ with the maximum of about $2 \mathrm{Mb}$, which agrees very well with the experiments. Further investigation found that this is a $\sigma_{u}^{*}$-type resonance, which is found in the components with a total $\Sigma_{u}$ symmetry after photoionization. The main contribution comes from the channel leading to the $1 \sigma_{g}^{-1}$ ion state. By looking only at the components with final $\Sigma_{u}$ symmetry, the single-channel calculations show that the contribution from the channel of the final $1 \sigma_{u}^{-1}$ ion state is about $10 \%$ of the channel of the final $1 \sigma_{g}^{-1}$ ion state at the peak of the shape resonance, and, in the coupled-channel calculation, the ratio only slightly increases. The result apparently suggests that there is little 
interchannel coupling effects between these two channels, which contradicts the recent random phase approximation (RPA) results that suggest strong coupling between these two channels (Cherepkov et al., 2000). We also compare the calculations using the RCHF approximation and using the $\mathrm{CI}$ approximation. The coupled-channel calculations using the RCHF approximation also suggest much stronger interchannel coupling effects (Lin \& Lucchese, 2000b). In the study of $\mathrm{C} 1 s$ photoionization of $\mathrm{C}_{2} \mathrm{H}_{2}$, we have already found that neglecting the correlation of the target ion states leads to exaggerated interchannel coupling effects in the coupled-channel scattering calculation (Lin \& Lucchese, 2000a). Our results in the $\mathrm{N} 1 s$ photoionization study of $\mathrm{N}_{2}$ suggest that only the simple treatment of the core relaxation effect is not sufficient in such a highly correlated system.

New experimental developments also enable the measurement of the angular distribution of the photoelectron in the fixed-in-space molecular frame, which provides extra data and information about the dynamics of the core photoionization process (Cherepkov et al., 2000). Theoretical studies are in progress to calculate such angular distributions, and also to include the shake-up channels in the closecoupling expansion. Thus, we will be able to better understand the electron correlation effects in the photoionization process, and to study the interchannel couplings between core-hole states and shakeup channels.

\section{Conclusions}

In this paper we studied the $\mathrm{N} 1 s$ photoionization of $\mathrm{NO}$ and $\mathrm{N}_{2}$. There are several significant differences between these two processes.

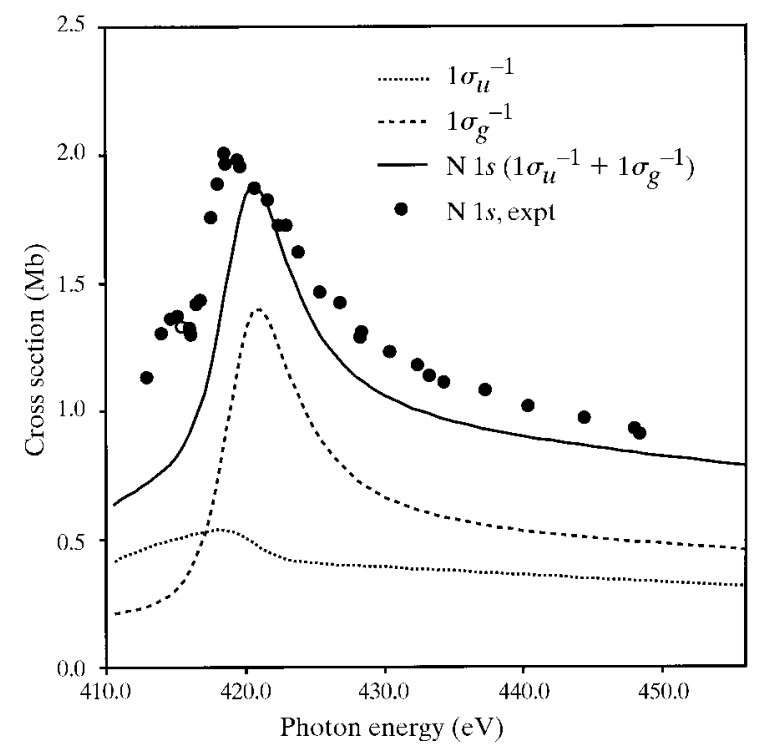

\section{Figure 2}

Total photoionization cross sections for ionization of $\mathrm{N}_{2}$ leading to the $\mathrm{N} 1 s^{-1}$ states of $\mathrm{N}_{2}^{+}$. The computed cross sections were obtained from a $2 \mathrm{CSCI}$ calculation. The experimental data are from Kempgens et al. (1996).
First of all, $\mathrm{N}_{2}$ is a close-shell molecule while NO is an open-shell molecule. The $\mathrm{N} 1 s$ ionization of NO leads to target ion states with different multiplicities, and NO will have more channels leading to the target states due to different spin couplings. Also, $\mathrm{N}_{2}$ is a homonuclear molecule; both $\mathrm{N}$ atoms contribute to the $\mathrm{N} 1 s$ photoionization. Thus, there are more channels coming from the initial state of $\mathrm{N}_{2}$. The two molecules have different symmetry properties, which lead to different coupling and transition rules. Their photoionization spectra also show distinct and interesting characters of the dynamic processes.

Besides these differences, we also observed some similarities in the $\mathrm{N} 1 s$ photoionization. Both the $\mathrm{N}_{2}$ and $\mathrm{NO}$ molecules have shape resonances located near the threshold, which have the character of the antibonding orbital. We also find that there are only small couplings between channels of different final core-hole states when properly correlated ion states are used in the calculations.

We acknowledge support of this work by the Welch Foundation (Houston) under grant number A-1020. We also acknowledge the support of the Texas A\&M University Supercomputing Facility.

\section{References}

Bandarage, G. \& Lucchese, R. R. (1993). Phys. Rev. A, 47, 1989-2003. Botting, S. K. \& Lucchese, R. R. (1997). Phys. Rev. A, 56, 3666-3674.

Chen, C. T., Ma, Y. \& Sette, F. (1989). Phys. Rev. A, 40(11), 6737-6740

Cherepkov, N. A., Semenov, S. K., Hikosaka, Y., Ito, K., Motoki, S. \& Yagishita, A. (2000). Phys. Rev. Lett. 84(2), 250-253.

Dehmer, J. L. \& Dill, D. (1975). Phys. Rev. Lett. 35, 213-215.

Domcke, W. \& Cederbaum, L. S. (1977). Chem. Phys. 25, 189-196.

Dunning, T. H. Jr (1989). J. Chem. Phys. 90(2), 1007-1023.

Hinze, J. (1981). Editor. The Unitary Group for the Evaluation of Electronic Energy Matrix Elements. Lecture Notes in Chemistry. Berlin: Springer.

Kempgens, B., Kivimäki, A., Neeb, M., Köppe, H. M., Bradshaw, A. M. \& Feldhaus, J. (1996). J. Phys. B, 29, 5389-5402.

Lin, P. \& Lucchese, R. R. (2000a). J. Chem. Phys. 113(5), 1843-1851.

Lin, P. \& Lucchese, R. R. (2000b). In preparation.

Lucchese, R. R. \& McKoy, V. (1983). Phys. Rev. A, 28, 1382-1394.

Lucchese, R. R., Raseev, G. \& McKoy, V. (1982). Phys. Rev. A, 25(5), 2572 2587.

Lucchese, R. R., Takatsuka, K. \& McKoy, V. (1986). Phys. Rep. 131(3), 147221.

Lynch, D. L. \& McKoy, V. (1984). Phys. Rev. A, 30(3), 1561-1564.

Natalense, A. P. P. \& Lucchese, R. R. (1999). J. Chem. Phys. 111(12), 5344 5348.

Rescigno, T. N. \& Langhoff, P. W. (1977). Chem. Phys. Lett. 51(1), 65-70.

Ruedel, A. \& Hergenhahn, U. (2000). Private communication.

Siegbahn, K., Nordling, C., Johansson, G., Hedman, J., Hedin, P. F., Hamrin, K., Gelius, U., Bergmark, T., Werme, L. O., Manne, R. \& Baer, Y. (1969). ESCA Applied to Free Molecules. Amsterdam: North-Holland.

Stratmann, R. E. \& Lucchese, R. R. (1995). J. Chem. Phys. 102(21), 8493-8505.

Stratmann, R. E., Zurales, R. W. \& Lucchese, R. R. (1996). J. Chem. Phys. 104(22), 8989-9000.

Thomas, T. D., Berrah, N., Bozek, J., Carroll, T. X., Hahne, J., Karlsen, T., Kukk, E. \& Saethre, L. J. (1999). Phys. Rev. Lett. 82(6), 1120-1123.

Wallace, S., Dill, D. \& Dehmer, J. L. (1982). J. Chem. Phys. 76(3), 1217-1222.

Wells, M. \& Lucchese, R. R. (1999). J. Chem. Phys. 110(13), 6365-6380.

Werner, H.-J. \& Knowles, P. J. (1996). MOLPRO. University of Birmingham, UK. 